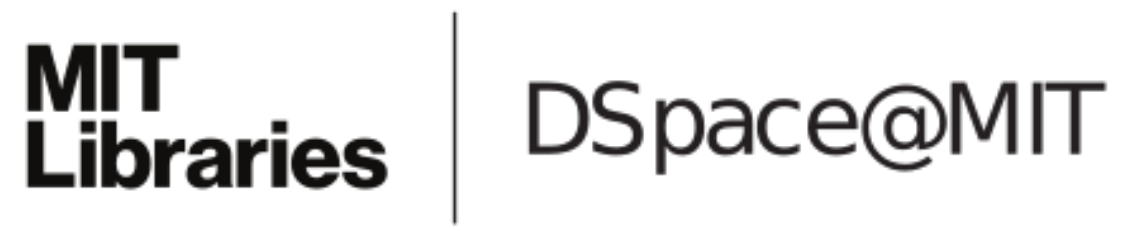

\author{
MIT Open Access Articles
}
Group-III quantum defects in diamond are stable spin-1 color centers

The MIT Faculty has made this article openly available. Please share how this access benefits you. Your story matters.

Citation: Harris, Isaac et al. "Group-III quantum defects in diamond are stable spin-1 color centers." Physical Review B, 102, 15 (November 2020): 195206 () 2020 The Author(s)

As Published: 10.1103/PhysRevB.102.195206

Publisher: American Physical Society (APS)

Persistent URL: https://hdl.handle.net/1721.1/129581

Version: Final published version: final published article, as it appeared in a journal, conference proceedings, or other formally published context

Terms of Use: Article is made available in accordance with the publisher's policy and may be subject to US copyright law. Please refer to the publisher's site for terms of use. 


\title{
Group-III quantum defects in diamond are stable spin-1 color centers
}

\author{
Isaac Harris, ${ }^{1,2,{ }^{*}}$ Christopher J. Ciccarino,,${ }^{1,3,{ }^{*}}$ Johannes Flick, ${ }^{1}$ Dirk R. Englund, ${ }^{2}$ and Prineha Narang $\circledast^{1, \dagger}$ \\ ${ }^{1}$ John A. Paulson School of Engineering and Applied Sciences, Harvard University, Cambridge, Massachusetts 02138, USA \\ ${ }^{2}$ Department of Electrical Engineering and Computer Science, Massachusetts Institute of Technology, Cambridge, Massachusetts 02138, USA \\ ${ }^{3}$ Department of Chemistry and Chemical Biology, Harvard University, Cambridge, Massachusetts 02138, USA
}

(Received 17 August 2019; accepted 4 November 2020; published 24 November 2020)

\begin{abstract}
Color centers in diamond have emerged as leading solid-state "artificial atoms" for a range of quantum technologies, from quantum sensing to quantum networks. Concerted research activities are now underway to identify new color centers that combine stable spin and optical properties of the nitrogen vacancy $\left(\mathrm{NV}^{-}\right)$ with the spectral stability of the silicon vacancy $\left(\mathrm{SiV}^{-}\right)$centers in diamond, with recent research identifying other group-IV color centers with superior properties. In this paper, we investigate a class of diamond quantum emitters from first principles, the group-III color centers, which we show to be thermodynamically stable in a spin-1, electric-field-insensitive structure. From $a b$ initio electronic structure methods, we characterize the product Jahn-Teller (pJT) effect present in the excited-state manifold of these group-III color centers, where we capture symmetry-breaking distortions associated with strong electron-phonon coupling. These predictions can guide experimental identification of group-III vacancy centers and their use in applications in quantum information science and technology.
\end{abstract}

DOI: 10.1103/PhysRevB.102.195206

\section{INTRODUCTION}

Diamond color centers are promising building blocks for applications such as quantum sensing and quantum communication [1-4], as shown in the recent demonstration of the generation of entanglement between two distant diamond nitrogen vacancy $\left(\mathrm{NV}^{-}\right)$centers at a rate faster than their decoherence [5]. While this result marks a major milestone towards the development of scalable quantum networks, the entanglement generation rate could be improved by orders of magnitude with a high-efficiency photonic interface to the emitter's coherent optical transition. One route is to couple the $\mathrm{NV}^{-}$center to a micro- or nanocavity to enhance spontaneous emission into the coherent zero phonon line. However, more work is needed to overcome the $\mathrm{NV}^{-}$, s spectral diffusion near surfaces [6], which arises primarily from its $C_{3 v}$ symmetry causing a permanent electric dipole moment.

Another approach, which is considered here, is to develop alternative color centers that have stable optical and spin properties. Within the diamond material system, the groupIV vacancy centers $\left(\mathrm{SiV}^{-}, \mathrm{GeV}^{-}, \mathrm{SnV}^{-}\right.$, and $\left.\mathrm{PbV}^{-}\right)$have been characterized experimentally [7-14] and theoretically [15-17]. The group-IV negative centers adopt an inversionsymmetric split-vacancy structure which has no permanent electric dipole moment, making their optical transitions less sensitive to electric field noise near surfaces [18]. Additionally, the branching ratio into the zero phonon line (ZPL) can be more than an order of magnitude higher than for the $\mathrm{NV}$ center, though this gain is partially offset by a lower

\footnotetext{
*These authors contributed equally to this work.

†prineha@seas.harvard.edu
}

internal quantum efficiency. Unfortunately, the negatively charged group-IV centers suffer from a phonon-mediated dephasing mechanism of the ground-state spin structure, which limits their spin coherence times [19]. Recent findings suggest that by careful boron doping of diamond, the $\mathrm{SiV}$ can favor a neutral charge with the stable spin-1 electronic ground state of the $\mathrm{NV}^{-}$center and the stable optical transitions of the $\mathrm{SiV}^{-}$[20], though more work is needed to demonstrate both properties in the same emitter. A natural question is whether there are other color centers that exhibit stable optical and spin properties in their thermodynamically favored charge state in intrinsic diamond. Outside of the group-IV centers, previous work has characterized other dopant vacancy centers in diamond [21]; however their optoelectronic properties are not yet understood.

In this paper, we report predictions of the diamond groupIII vacancy defects $X \mathrm{~V}$, with $X=\mathrm{Al}, \mathrm{Ga}$, In, and $\mathrm{Tl}$, and characterize their properties using ab initio electronic structure theory of the ground- and excited-state manifolds. Our calculations reveal that the three lightest defects are stable in inversion-symmetric configurations, with a thermodynamically preferred -1 charge state which makes them isoelectronic with the $\mathrm{SiV}^{0}$ defect. We characterize the product Jahn-Teller (pJT) effect $[17,22]$ present in the excited-state manifold, where we capture symmetry-breaking distortions associated with strong electron-phonon coupling. While these distortions have a large effect on the zero-phonon line energies, they preserve the inversion symmetry in the excited state, maintaining the desired charge noise insensitivity. We also capture vibronic spectra and discuss the relatively high predicted ZPL emission efficiency found. Overall, the groupIII vacancy centers are found to be similar to their group-IV neutral counterparts, but with a spin- 1 ground state for the 

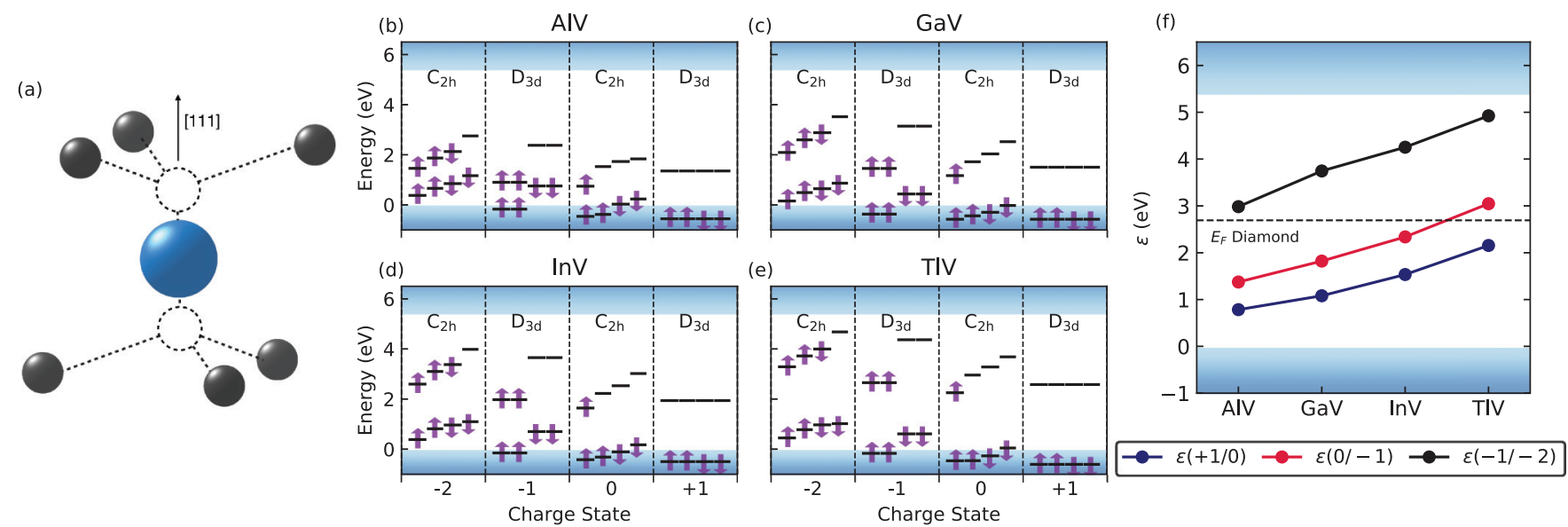

FIG. 1. (a) $\mathrm{D}_{3 \mathrm{~d}}$ ground-state structure of a group-III vacancy defect in diamond. The impurity atom lies directly between two carbon empty lattice sites, equidistant to six nearest-neighbor carbons. (b)-(e) Ground-state spin-resolved energy level structure of the group-III vacancies in charge states -2 to +1 . Both the -1 and +1 charge states are stable in the high-symmetry $\mathrm{D}_{3 \mathrm{~d}}$ structure, while the -2 and neutral charge states have uneven occupations and distort to a $\mathrm{C}_{2 \mathrm{~h}}$ structure. (f) Predicted thermodynamic charge transition levels for each of the group-III defect centers. The dashed line labeled " $E_{F}$ Diamond" is the Fermi level calculated for bulk, pristine diamond.

thermodynamically stable negative charge state in intrinsic diamond. The combination of the stable spin-1 ground state and symmetry-protected optical transitions makes these centers excellent candidates for quantum technologies.

\section{RESULTS AND DISCUSSION}

First, we investigate the electronic properties of the groupIII vacancies for aluminum, gallium, indium, and thallium using density functional theory (see the Appendix). All four substituents are found to energetically favor the split-vacancy complex shown in Fig. 1(a) over the separate substitutional and vacancy defects and are therefore expected to form under typical implantation and annealing conditions. Depending on the charge state, this configuration has either a $D_{3 d}$ geometry with the principal axis oriented along the [111] crystallographic direction or a $\mathrm{C}_{2 \mathrm{~h}}$ geometry. Both configurations are inversion symmetric about the substituent atom and therefore cannot have a net dipole moment, making their optical transitions insensitive to charge noise. Each of the presented calculations uses a 512-atom "supercell" and samples the $\Gamma$ point; we refer to the Appendix for computational details. $\mathrm{BV}^{-}$is not included in this work as previous work has shown its formation from B substitutional and vacancy defects is not as energetically favorable as the NV, group-IV elements, or other group-III elements [21]. Additionally, its small atomic size means it sits at a substitutional rather than the desirable interstitial site in the defect-vacancy complex [21], and it is therefore expected to behave in a significantly different way than the other group-III elements. Additionally, higher-order complexes are not considered as they are not likely to form due to low mobility of substitutional defects in diamond [23] at typical processing temperatures.

As previous work $[15-17,24]$ has established for group-IV emitters in diamond, the orbitals associated with the group-III defects can be identified by their symmetry and are composed of dangling carbon bonds and $s$ and $p$ orbitals from the dopant atom. We find two pairs of degenerate Kohn-Sham (KS) orbitals are localized within the diamond band gap, having $E_{u}$ and $E_{g}$ symmetry, respectively. The orbitals within each degenerate pair are denoted as $e_{u x(y)}$ and $e_{g x(y)}$, where $x$ or $y$ refers to their orientation. While additional localized defect orbitals are present, they are energetically far from the band edges and are therefore not accessible for optical excitation. The symmetry of these orbitals originates from the point group symmetry of the defect; thus, we find the same orbitals as for the group-IV defects.

Figure 1 shows the electronic ground-state configurations for these defects in charge states from -2 to +1 . Figures 1(b)1 (e) plot the energies of the spin-majority (spin-up) and spinminority (spin-down) channels for each of the defects, where the $e_{u}$ orbitals lie below the $e_{g}$ orbitals in each case.

Of particular interest are the -1 charge states, which are spin-1 states, isoelectronic to the group-IV neutrals [17] and stable in a $\mathrm{D}_{3 \mathrm{~d}}$ configuration. For each defect, the ground-state electronic configuration of the -1 charge state is denoted by $e_{u}^{4} e_{g}^{2}$, such that there are two unfilled $e_{g}$ orbitals, both of the same spin channel. This can equivalently be formulated in terms of a pair of holes occupying the remaining $e_{g}$ groundstate orbitals. In contrast to ab initio orbital energetics of group-IV neutral systems $[15,17]$, where the $e_{u}$ KS orbitals lie below the valence band edge, both the $e_{u}$ and $e_{g}$ levels for the spin-minority channel are located within the band gap for the group-III negatives. The isolation of the defect states from the bulk bands make single-photon transitions from the defect to bulk states less likely when addressing the defect-defect transition, potentially improving the excitation efficiency for the group-III vacancy centers.

Next, we calculate which charge state is thermodynamically most favorable at a given doping level for the diamond host. The Fermi level at which a transition becomes thermodynamically stable is given by the charge transition level (CTL) [25]:

$$
\varepsilon\left(q_{1} / q_{2}\right)=\frac{E_{q_{1}}^{\mathrm{tot}}+E_{q_{1}}^{\mathrm{corr}}-E_{q_{2}}^{\mathrm{corr}}-E_{q_{2}}^{\mathrm{tot}}}{q_{2}-q_{1}},
$$


with $E_{q}^{\text {tot }}$ being the total energy from the supercell calculation in charge state $q$ and $E_{q}^{\text {corr }}$ being the corresponding charge correction to account for periodic interaction of charges between neighboring supercells [26-28]. We apply the correction techniques described in Ref. [26], yielding the results summarized in Fig. 1(f).

Our calculated CTLs are useful in understanding the thermodynamic situations in which the desirable -1 charge state will be preferred. The -1 charge state of these defects would act as electron acceptors, which require compensatory electron donors present in the system. One common example of electron donors would be nitrogen substitutions, which have a deep donor level $1.7 \mathrm{eV}$ below the conduction band maximum [29-31]. This nitrogen doping would shift the Fermi level higher towards the conduction band edge. In the case of InV and TlV, this positive Fermi energy shift may improve the stability of the $q=-1$ charge state. For $\mathrm{GaV}$ and especially for AlV, the thermodynamic stability window is at a slightly lower Fermi energy. The $q=-1$ charge state may still be realized; however, too much $n$-type doping may push these emitters to the $q=-2$ charge state instead. We note that other electron donors in the system such as the $\mathrm{NV}^{-}$or boron substitutions would pull the Fermi level closer to the valence band edge, which may improve stability of $\mathrm{GaV}^{-}$and $\mathrm{AlV}^{-}$. In general it is difficult to pinpoint the exact location of the Fermi level given that it is sample dependent and depends on all of the impurities and defects in the crystal of interest.

We note, however, that our calculated CTLs are computed in a way similar to the CTLs for group-IV defects [15]. These CTL predictions of the group-III and group-IV emitters are very similar and have had much success in predicting the charge dynamics of group-IV emitters compared to experiment. While experimental investigations of group-III defects have not been reported, the similarity in the CTL predictions along with the success of these methods for group-IV defect charge behavior indicates that the stability of the charge state associated with a $S=1$ spin state is more likely to be found in group-III emitters for typical diamond samples. The Fermi levels associated with the stability of the $S=1$ charge state (i.e., $q=-1$ for group III and $q=0$ for group IV) are higher for the group-III defects we study here. In the case of the $\mathrm{SiV}^{0}$, achieving the $S=1$ charge state required Boron doping, i.e., lowering the Fermi level. The higher Fermi energy window associated with the group-III negatives studied here suggests these candidates may be found without the need for lowering the Fermi energy or doping in general.

Given that these color centers are isoelectronic with the $\mathrm{SiV}^{0}$, the negatively charged group-III vacancy centers are expected to have electronic properties similar to the $\mathrm{SiV}^{0}$ in the ground and excited states. In particular, exciting an electron from the lower-energy $e_{u}$ to the higher-energy $e_{g}$ orbital means that the electronic configuration becomes $e_{u}^{3} e_{g}^{3}$, producing an unequal occupation of the two degenerate orbital pairs. Such a system is expected to be Jahn-Teller unstable [32] as a result of a product of two the Jahn-Teller instabilities for both the $e_{u}$ and $e_{g}$ orbitals, denoted as a $\left(e_{g} \otimes e_{u}\right) \otimes E_{g}$ product Jahn-Teller effect [17]. Here $E_{g}$ is the irreducible representation of the coupled phonon modes that produce the distortion. Each of these modes is twofold degenerate, and the Jahn-Teller distortion therefore resides in a two-dimensional coordinate space that contains the high-symmetry $\mathrm{D}_{3 \mathrm{~d}}$ point, as well as three equivalent energy minima in the $\mathrm{C}_{2 \mathrm{~h}}$ symmetry group. Accurately describing the Jahn-Teller effect is important in predicting the experimentally relevant zero phonon line energy associated with optical emission, as the pJT symmetry reduction can result in a large energy change in the excited state, as seen in the case of the $\mathrm{SiV}^{0}[17,20,33]$.

To capture this effect in group-III vacancy centers, we relax the $\mathrm{GaV}^{-}, \mathrm{InV}^{-}$, and $\mathrm{TlV}^{-}$defects in the excited electronic state using constrained density functional theory (DFT; $\Delta$ self-consistent field (SCF), see the Appendix). First, we relax the system constrained to both $\mathrm{D}_{3 \mathrm{~d}}$ and $\mathrm{C}_{2 \mathrm{~h}}$ geometries with excited electronic occupations until forces on all atoms are below $10^{-6} \mathrm{Ry} /$ bohr. We additionally verify that the resulting $\mathrm{C}_{2 \mathrm{~h}}$ structure is the true minimum of the excited-state manifold by relaxing without any symmetry constraints. From these relaxed $\mathrm{C}_{2 \mathrm{~h}}$ and $\mathrm{D}_{3 \mathrm{~d}}$ geometries, we can construct the full-potential energy surface associated with the Jahn-Teller modes in the excited manifold. $\mathrm{AlV}^{-}$is omitted from the excited-state calculations due to numerical convergence challenges with the system. The small energy difference between the $e_{u}$ and $e_{g}$ states causes oscillations of the occupations during iterative minimization, which prevented us from obtaining its excited-state properties. However, it is expected to follow the same trends as the heavier group-III elements.

Similar to the behavior of the group-IV neutral excited states in Ref. [17], four distinct excited states may exist depending on the occupations of the defect orbitals, as can be seen from the fact that the two holes can each occupy two orbitals. However, our current techniques are able to access only the lowest excited-state energy configurations for a given geometry; therefore, we consider only the lowest of the two excited-state potential energy surfaces. Future extensions of this work could use a formalism along the lines of Refs. [34,35], which restrict the symmetry of the orbitals, allowing for a better approximation of the many-body Hamiltonian and a slightly more accurate ZPL energy calculation. In the case of group-IV neutrals, these many-body effects are found to shift the ZPL energy by less than $0.03 \mathrm{eV}(\sim 2 \%)$ from the energy difference obtained from DFT. While these differences might be slightly different for the group-III centers studied here, they are still expected to be of the same order of magnitude, so we choose to approximate the ZPL energy without considering these additional many-body effects. We also note that a deeper understanding of the excited-state level structure can be achieved by considering additional physical effects such as spin-orbit coupling [36] or by using alternative computational methods, such as $G W$ [37] or time-dependent DFT [38]. However, our results provide an important first step towards characterizing these emitters. The calculated ZPL energies, given as the energy difference between the $\mathrm{C}_{2 h}$ excited electronic state and the $\mathrm{D}_{3 \mathrm{~d}}$ ground electronic state, are summarized in Table I.

Figure 2 and Table I summarize the results of the pJT calculations. Figure 2(a) shows a one-dimensional interpolation of the excited-state potential energy surface between the $\mathrm{D}_{3 \mathrm{~d}}$ (located at $Q=0$ ) and $\mathrm{C}_{2 \mathrm{~h}}$ geometries. The global minimum is the relaxed $\mathrm{C}_{2 \mathrm{~h}}$ geometry, while the local minimum on the opposite side of the $\mathrm{D}_{3 \mathrm{~d}}$ point is a saddle point. The energy differences between the $\mathrm{D}_{3 \mathrm{~d}}$ and global $\mathrm{C}_{2 \mathrm{~h}}$ minima $(\Delta)$ and 
TABLE I. Energetics of group-III color centers $\mathrm{GaV}^{-}, \mathrm{InV}^{-}$, and $\mathrm{TIV}^{-}$. The predicted Jahn-Teller instabilities, depicted in Fig. 2, are given by $\Delta$ and $\delta$. The zero phonon line energies are given by $E_{\mathrm{ZPL}}$ (see Fig. 3).

\begin{tabular}{lccc}
\hline \hline & $\Delta(\mathrm{meV})$ & $\delta(\mathrm{meV})$ & $E_{\mathrm{ZPL}}(\mathrm{nm}, \mathrm{eV})$ \\
\hline $\mathrm{GaV}^{-}$ & 236 & 71 & $679,1.82$ \\
$\mathrm{InV}^{-}$ & 175 & 60 & $584,2.12$ \\
$\mathrm{TlV}^{-}$ & 148 & 58 & $437,2.84$ \\
\hline \hline
\end{tabular}

the saddle point and global $\mathrm{C}_{2 \mathrm{~h}}$ minima $(\delta)$ are presented in Table I. In the first-order Jahn-Teller effect, the potential energy surface would be symmetrical with respect to the $D_{3 d}$ point, such that $\delta \rightarrow 0$. However, given the relatively large values of $\delta$, higher-order effects are clearly important in this system [32]. We note that these $\delta$ values are consistent in
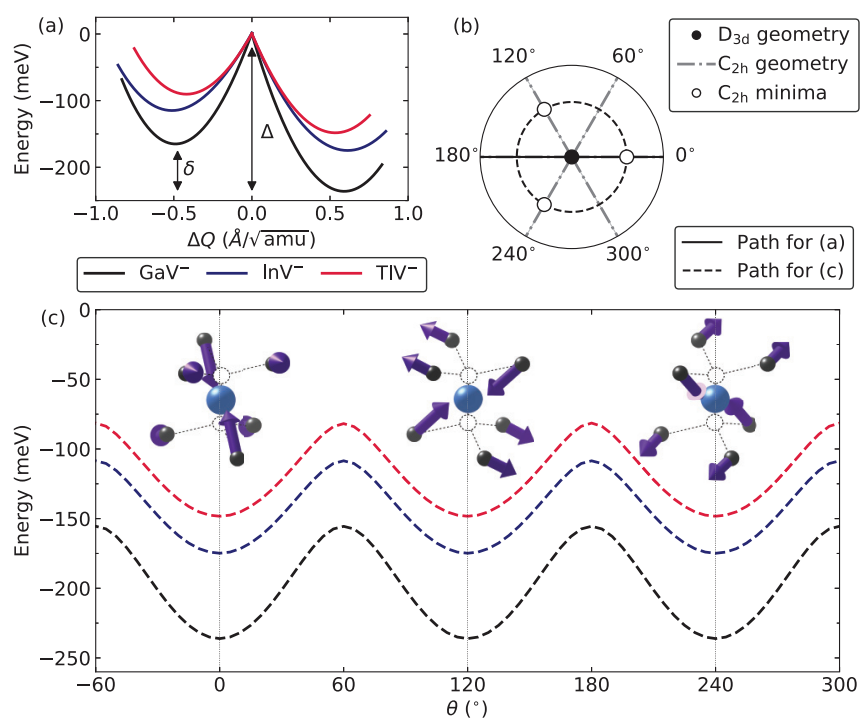

FIG. 2. Product Jahn-Teller instability for group-III vacancy centers $\mathrm{GaV}^{-}, \mathrm{InV}^{-}$and $\mathrm{TlV}^{-}$. (a) Linear interpolation between the relaxed low-symmetry $\mathrm{C}_{2 \mathrm{~h}}$ and high-symmetry $\mathrm{D}_{3 \mathrm{~d}}$ geometries for the excited-state electronic configuration $e_{u}^{3} e_{g}^{3}$. The distortion is favored due to a product Jahn-Teller effect caused by the occupational imbalances in the $e_{u}$ and $e_{g}$ orbitals. The computed instability energies are given as $\Delta$ and listed in Table I, where we also list the energy barrier $\delta$ caused by higher-order phonon coupling. (b) The configuration spaces plotted in (a) and (c) are depicted relative to the high-symmetry $D_{3 d}$ (black dot) and three low-symmetry $C_{2 h}$ points (white dots). The solid (dashed) black line indicates the path taken along the $x$ axis for (a) [(c)]. The gray dot-dashed lines indicate the areas where the defect has $\mathrm{C}_{2 \mathrm{~h}}$ symmetry, with all points not on the lines (and not at the center $\mathrm{D}_{3 \mathrm{~d}}$ point) having $\mathrm{C}_{\mathrm{i}}$ symmetry. (c) Calculated energies for a path equidistant to the high-symmetry $D_{3 \mathrm{~d}}$ point shown in (b), where we find three energetically equivalent $C_{2 h}$ geometries. The local minima represent saddle points, corresponding to the local minima shown in (a). Additionally pictured are the three equivalent distortions from $D_{3 d}$ to each of the $C_{2 h}$ minima. Purple arrows in the inset indicate the magnitude and direction of distortions of the six nearest-neighbor atoms for the three equivalent Jahn-Teller minima. magnitude with values obtained for group-IV neutral emitters $[17,36]$.

In Fig. 2(c), we map out the energy of the system along a circle through the Jahn-Teller coordinate space which traverses the three energetically equivalent $C_{2 h}$ minimum geometries, as well as the energy barriers between them caused by the higher-order Jahn-Teller coupling. The trajectories in the coordinate space shown in Figs. 2(a) and 2(c) are visualized in Fig. 2(b), where we depict the locations of highsymmetry $D_{3 d}$ geometry along with the three equivalent $C_{2 h}$ configurations within the JT space. The movements of the nearest-neighbor carbons resulting from each of the equivalent distortions are shown as well. We note that each distortion affects one pair of the nearby carbon atoms more than the other two. By adding each of these distortions together, we arrive at the high-symmetry $\mathrm{D}_{3 \mathrm{~d}}$ geometry once again.

To characterize the optical properties, we calculate $a b$ initio emission line shapes using the method outlined in Ref. [39] (see the Appendix). The emission line shape can then be calculated from the overlap of the ionic vibrational wave functions between the relaxed excited electronic state and the electronic ground vibrational states $[39,40]$ according to

$$
A(\hbar \omega)=\sum_{m}\left|\left\langle\chi_{\mathrm{e} 0} \mid \chi_{\mathrm{g} m}\right\rangle\right|^{2} \delta\left(E_{\mathrm{ZPL}}-E_{\mathrm{g} m}-\hbar \omega\right),
$$

where $\chi_{\mathrm{g}(\mathrm{e}) m}$ is the $m$ th vibrational state of the ground (excited) electronic state and $\omega$ is the frequency of the transition. $E_{\mathrm{g} m}$ represents the energy of the vibrational state $\chi_{\mathrm{g} m}$. The true emission line shape is determined by Fermi's golden rule and is proportional to this overlap and the transition frequency as $L(\hbar \omega) \propto \omega^{3} A(\hbar \omega)$.

The vibrational overlaps are evaluated using a generating function approach as described previously [39]. The generating function method assumes that the modes in the ground and excited states are identical, and there is only a difference in geometry between the two configurations. This assumption is strongly violated when a Jahn-Teller distortion occurs in the excited state since the symmetries between the two geometries are different. Using the $\mathrm{C}_{2 \mathrm{~h}} \rightarrow \mathrm{D}_{3 \mathrm{~d}}$ transition therefore underestimates the overlap of the ground vibrational states in the ground- and excited-state electronic manifolds. Previous theoretical approaches for generating emission spectra for JTunstable defects in diamond have neglected the effect of the JT distortion by equally occupying the degenerate electronic orbitals using fractional occupations, effectively enforcing a high-symmetry configuration (e.g., in the $\mathrm{NV}^{-}$[39] and $\mathrm{SiV}^{-}$ [41]). We use a similar approximation by studying the phonon coupling to the emission from the excited state with the correct orbital occupations, but restricted to a $\mathrm{D}_{3 \mathrm{~d}}$ geometry. We note that this leads to a slightly lower Debye-Waller factor than the fractional occupation technique for the excited electronic states.

Figure 3 summarizes the optical properties of the group-III defects. Figure 3(a) shows ZPL energies, which are comparable to the equivalent group-IV defects (which have ZPLs

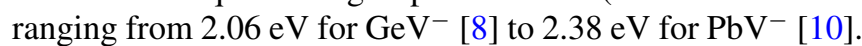
The line shapes in Fig. 3(c) give information on what to expect from photoluminescence experiments. The Debye-Waller factor in Fig. 3(b), which describes the overall emission efficiency into the ZPL, is an important figure of merit for 

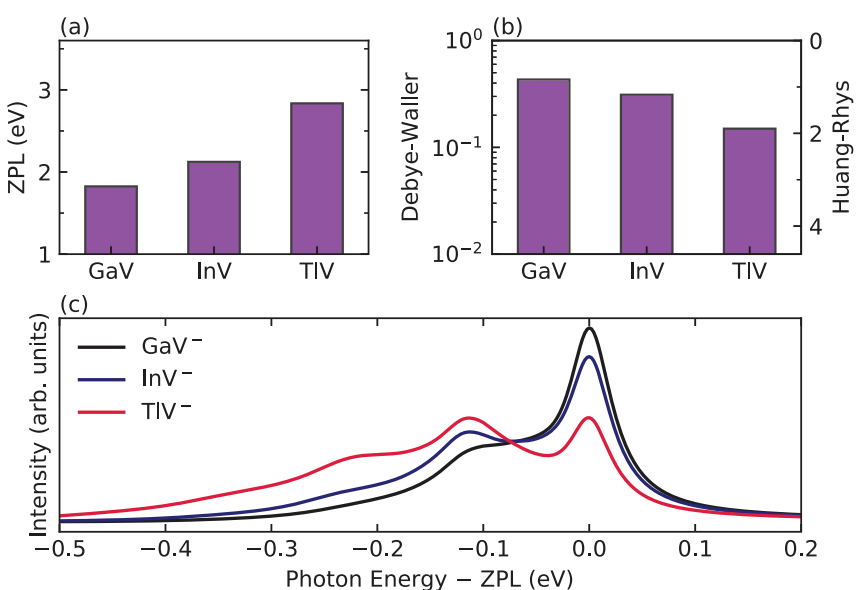

FIG. 3. (a) Predicted zero phonon line energy for each of the color centers found using $\triangle \mathrm{SCF}$, including the $\mathrm{pJT}$ energy reduction in the excited state. (b) Debye-Waller factor (left) and Huang-Rhys factor (right) for each color center. The Debye-Waller factor indicates the fraction of photons emitted into the ZPL, while the Huang-Rhys factor indicates the mean number of phonons emitted with each photon. (c) Ab initio spectra for the $\mathrm{D}_{3 \mathrm{~d}} \rightarrow \mathrm{D}_{3 \mathrm{~d}}$ transition showing the predicted emission line shape on a linear scale relative to the zero phonon line. We include linewidth broadening associated with near room temperature conditions.

the performance of these defects in quantum protocols such as photon-mediated entanglement [3]. The resulting spectra, along with the predicted Huang-Rhys and Debye-Waller factors, are given in Fig. 3. The emission efficiency into the $\mathrm{ZPL}$ is much higher than what is observed in $\mathrm{NV}^{-}$[42], although we find that the efficiency decreases for heavier defect elements.

\section{SUMMARY}

In conclusion, we predicted the quantum optoelectronic properties of diamond group-III vacancy centers. Our calculations showed that these color centers are stable in intrinsic diamond in an $S=1$ spin state with a symmetry that makes their transitions insensitive to electric fields. While we did not explicitly calculate properties of the ground-state spin manifold such as zero- field splitting and the effective $g$ factor, the similarities of the orbital shapes and structures we find between the group-III defects and the group-IV neutrals suggest these parameters will be of a similar order of magnitude. As has been shown experimentally for the $\mathrm{SiV}^{0}$, we expect that these group-III defects are likely to have favorable spin coherence properties [20]; however, further work is needed to verify this. The combination of favorable thermodynamics, stable spin, and symmetry-protected optical properties makes the negatively charged group-III vacancy centers ideal candidates for applications such as quantum networking and computing. The emerging ability to perform detailed calculations on spin and optical properties of nanoscopic quantum emitters, including corrections such as the large-product JahnTeller effect, marks an important step towards ab initio design and discovery of quantum materials [43]. In particular, the predicted properties of the group-III vacancy class of emitters would combine the desired attributes of high Debye-Waller factors with highly coherent spin and optical transitions at a range of wavelengths, promising a new class of quantum emitters for applications in quantum information science and technology.

\section{ACKNOWLEDGMENTS}

We thank Dr. M. Trusheim (MIT-Harvard) for helpful discussions. This work was supported by the DOE "Photonics at Thermodynamic Limits" Energy Frontier Research Center under Grant No. DE-SC0019140. D.R.E. and P.N. are partially supported by the Army Research Office MURI (Ab-Initio Solid-State Quantum Materials) Grant No. W911NF-18-1-0431. J.F. acknowledges fellowship support from the Deutsche Forschungsgemeinschaft (DFG) under Contract No. FL 997/1-1. P.N. acknowledges support as a Moore Inventor Fellow under Grant Number GBMF 8048. Calculations were performed using resources from the Department of Defense High Performance Computing Modernization program. Additional calculations were performed using resources of the National Energy Research Scientific Computing Center (NERSC), a U.S. Department of Energy Office of Science User Facility operated under Contract No. DE-AC02-05CH11231 as well as resources at the Research Computing Group at Harvard University.

\section{APPENDIX: METHODS}

All calculations were formed using the Kohn-Sham, planewave density functional theory code QUANTUM ESPRESSO $[44,45]$. The exchange-correlation interactions were approximated by the hybrid Heyd-Scuseria-Ernzerhof (HSE06) $[46,47]$ functional, which has been shown to describe the energetics of bulk diamond and other known diamond color centers with high accuracy. The group-III defects are described within a 512-atom supercell of diamond, built using the relaxed HSE06 lattice constant of the primitive cell, which is found to be $a=3.545 \AA$. We describe the system by sampling only at the $\Gamma$ point of the Brillouin zone. The KohnSham states are described using a plane-wave basis set with an energy cutoff of $80 \mathrm{Ry}$, and norm-conserving pseudopotentials [48] are used to describe the electronic interaction with the nuclei. In all instances, spin polarization is included.

When describing the luminescence line shape (i.e., Fig. 3 of the main text), we modeled the ground-state phonons of the system using a finite-difference approach as implemented in PHONOPY [49]; here we use the efficient Perdew-Burke-Ernzerhof (PBE) functional [50] with ultrasoft pseudopotentials [51]. The use of ultrasoft pseudopotentials gives the same results as the previously used norm-conserving pseudopotentials within numerical accuracy. Phonon properties computed using the PBE functional have previously been found to be sufficiently accurate compared to the hybrid functional used earlier [52]. However, both the ground- and excited-state geometries associated with the line shape correspond to those calculated using the HSE06 functional. 
[1] F. Jelezko and J. Wrachtrup, Phys. Status Solidi A 203, 3207 (2006).

[2] J. R. Weber, W. F. Koehl, J. B. Varley, A. Janotti, B. B. Buckley, C. G. Van de Walle, and D. D. Awschalom, Proc. Natl. Acad. Sci. USA 107, 8513 (2010).

[3] I. Aharonovich, D. Englund, and M. Toth, Nat. Photonics 10, 631 (2016).

[4] D. D. Awschalom, R. Hanson, J. Wrachtrup, and B. B. Zhou, Nat. Photonics 12, 516 (2018).

[5] P. C. Humphreys, N. Kalb, J. P. J. Morits, R. N. Schouten, R. F. L. Vermeulen, D. J. Twitchen, M. Markham, and R. Hanson, Nature (London) 558, 268 (2018).

[6] M. Atatüre, D. Englund, N. Vamivakas, S.-Y. Lee, and J. Wrachtrup, Nat. Rev. Mater. 3, 38 (2018).

[7] E. Neu, C. Hepp, M. Hauschild, S. Gsell, M. Fischer, H. Sternschulte, Doris Steinmüller-Nethl, M. Schreck, and C. Becher, New J. Phys. 15, 43005 (2013).

[8] T. Inubushi, N. Mizuochi, F. Ishibashi, M. Hatano, Y. Doi, Y. Miyamoto, L. J. Rogers, S. Kobayashi, S. Yamasaki, F. Jelezko, S. Nagamachi, B. Naydenov, K. Tahara, K. D. Jahnke, T. Iwasaki, and T. Miyazaki, Sci. Rep. 5, 12882 (2015).

[9] T. Iwasaki, Y. Miyamoto, T. Taniguchi, P. Siyushev, M. H. Metsch, F. Jelezko, and M. Hatano, Phys. Rev. Lett. 119, 253601 (2017).

[10] M. E. Trusheim, N. H. Wan, K. C. Chen, C. J. Ciccarino, J. Flick, R. Sundararaman, G. Malladi, E. Bersin, M. Walsh, B. Lienhard, H. Bakhru, P. Narang, and D. Englund, Phys. Rev. B 99, 075430 (2019).

[11] M. K. Bhaskar, D. D. Sukachev, A. Sipahigil, R. E. Evans, M. J. Burek, C. T. Nguyen, L. J. Rogers, P. Siyushev, M. H. Metsch, H. Park, F. Jelezko, M. Lončar, and M. D. Lukin, Phys. Rev. Lett. 118, 223603 (2017).

[12] D. D. Sukachev, A. Sipahigil, C. T. Nguyen, M. K. Bhaskar, R. E. Evans, F. Jelezko, and M. D. Lukin, Phys. Rev. Lett. 119, 223602 (2017).

[13] R. E. Evans, M. K. Bhaskar, D. D. Sukachev, C. T. Nguyen, A. Sipahigil, M. J. Burek, B. Machielse, G. H. Zhang, A. S. Zibrov, E. Bielejec, H. Park, M. Lončar, and M. D. Lukin, Science 362, 662 (2018).

[14] M.-A. Lemonde, S. Meesala, A. Sipahigil, M. J. A. Scheutz, M. D. Lukin, M. Loncar, and P. Rabl, Phys. Rev. Lett. 120, 213603 (2018).

[15] G. Thiering and A. Gali, Phys. Rev. X 8, 021063 (2018).

[16] A. Gali and J. R. Maze, Phys. Rev. B 88, 235205 (2013).

[17] G. Thiering and A. Gali, npj Comput. Mater. 5, 18 (2019).

[18] A. Sipahigil, K. D. Jahnke, L. J. Rogers, T. Teraji, J. Isoya, A. S. Zibrov, F. Jelezko, and M. D. Lukin, Phys. Rev. Lett. 113, 113602 (2014).

[19] M. W. Doherty, M. Metsch, N. B. Manson, M. D. Lukin, F. Jelezko, A. Sipahigil, K. D. Jahnke, L. J. Rogers, and J. M. Binder, New J. Phys. 17, 043011 (2015).

[20] B. C. Rose, D. Huang, Z.-H. Zhang, P. Stevenson, A. M. Tyryshkin, S. Sangtawesin, S. Srinivasan, L. Loudin, M. L. Markham, A. M. Edmonds, D. J. Twitchen, S. A. Lyon, and N. P. de Leon, Science 361, 60 (2018).

[21] J. P. Goss, P. R. Briddon, M. J. Rayson, S. J. Sque, and R. Jones, Phys. Rev. B 72, 035214 (2005).

[22] Q. C. Qiu and A. Ceulemans, in Vibronic Interactions: JahnTeller Effect in Crystals and Molecules, edited by M. D.
Kaplan and G. O. Zimmerman (Springer, Dordrecht, 2001), pp. 97-102.

[23] A. Mainwood, Phys. Rev. B 49, 7934 (1994).

[24] C. Hepp, T. Müller, V. Waselowski, J. N. Becker, B. Pingault, H. Sternschulte, D. Steinmüller-Nethl, A. Gali, J. R. Maze, M. Atatüre, and C. Becher, Phys. Rev. Lett. 112, 036405 (2014).

[25] C. Freysoldt, B. Grabowski, T. Hickel, J. Neugebauer, G. Kresse, A. Janotti, and C. G. Van de Walle, Rev. Mod. Phys. 86, 253 (2014).

[26] R. Sundararaman and Y. Ping, J. Chem. Phys. 146, 104109 (2017).

[27] C. Freysoldt, J. Neugebauer, and C. G. Van de Walle, Phys. Rev. Lett. 102, 016402 (2009).

[28] A. Goyal, K. Mathew, R. G. Hennig, A. Chernatynskiy, C. R. Stanek, S. T. Murphy, D. A. Andersson, S. R. Phillpot, and B. P. Uberuaga, App. Sci. 9, 5276 (2019).

[29] A. Mainwood, J. Phys. C 12, 2543 (1979).

[30] K. Kobashi, arXiv:1406.6204.

[31] A. T. Collins, J. Phys.: Condens. Matter 14, 3743 (2002).

[32] H.-H. Schmidtke, Ber. Bunsengesellschaft Phys. Chem. 94, 896 (1990).

[33] B. L. Green, M. W. Doherty, E. Nako, N. B. Manson, U. F. S. D'Haenens-Johansson, S. D. Williams, D. J. Twitchen, and M. E. Newton, Phys. Rev. B 99, 161112(R) (2019).

[34] B. Kaduk, T. Kowalczyk, and T. Van Voorhis, Chem. Rev. 112, 321 (2012).

[35] C. P. Plaisance, R. A. van Santen, and K. Reuter, J. Chem. Theory Comput. 13, 3561 (2017).

[36] C. J. Ciccarino, J. Flick, I. B. Harris, M. E. Trusheim, D. R. Englund, and P. Narang, npj Quantum Mater. 5, 75 (2020).

[37] Y. Ma, M. Rohlfing, and A. Gali, Phys. Rev. B 81, 041204(R) (2010).

[38] A. Gali, Phys. Status Solidi B 248, 1337 (2011).

[39] A. Alkauskas, B. B. Buckley, D. D. Awschalom, and C. G. Van De Walle, New J. Phys. 16, 073026 (2014).

[40] A. Alkauskas, J. L. Lyons, D. Steiauf, and C. G. Van de Walle, Phys. Rev. Lett. 109, 267401 (2012).

[41] E. Londero, G. Thiering, L. Razinkovas, A. Gali, and A. Alkauskas, Phys. Rev. B 98, 035306 (2018).

[42] M. W. Doherty, N. B. Manson, P. Delaney, F. Jelezko, J. Wrachtrup, and L. C. L. Hollenberg, Phys. Rep. 528, 1 (2013).

[43] H. Seo, H. Ma, M. Govoni, and G. Galli, Phys. Rev. Materials 1, 075002 (2017).

[44] P. Giannozzi et al., J. Phys.: Condens. Matter 21, 395502 (2009).

[45] P. Giannozzi et al., J. Phys.: Condens. Matter 29, 465901 (2017).

[46] J. Heyd, G. E. Scuseria, and M. Ernzerhof, J. Chem. Phys. 118, 8207 (2003).

[47] J. Heyd, G. E. Scuseria, and M. Ernzerhof, J. Chem. Phys. 124, 219906 (2006).

[48] M. Schlipf and F. Gygi, Comput. Phys. Commun. 196, 36 (2015).

[49] A. Togo and I. Tanaka, Scr. Mater. 108, 1 (2015).

[50] J. P. Perdew, K. Burke, and M. Ernzerhof, Phys. Rev. Lett. 77, 3865 (1996).

[51] K. F. Garrity, J. W. Bennett, K. M. Rabe, and D. Vanderbilt, Comput. Mater. Sci. 81, 446 (2014).

[52] K. Hummer, J. Harl, and G. Kresse, Phys. Rev. B 80, 115205 (2009). 